\title{
ESTIMACIÓN DEL COEFICIENTE DE CONSANGUINIDAD DE TOROS BROWN SWISS REGISTRADOS EN EL PERÚ, AÑO 2003
}

\author{
Estimation of Inbreeding Coefficient in Registered Brown Swiss Bulls \\ of Peru, Year 2003
}

\author{
Juan Oré C. ${ }^{1}$, Eduardo Fuentes N. ${ }^{1}$ y Edwin Mellisho S. ${ }^{1,2}$
}

\section{Resumen}

La consanguinidad puede reducir la fertilidad, salud y productividad de vacas lecheras y otras especies zootécnicas. El presente estudio tuvo como finalidad estimar el coeficiente de consanguinidad de los toros de la raza Brown Swiss registrados en el año 2003, y establecer el origen de sus padres y abuelos maternos. Se analizaron registros de 4 generaciones de 113 machos pedigrí registrados en la Asociación Brown Swiss del Perú el año 2003, los cuales incluyeron 3616 certificados de registro de pedigrí. Para la determinación del coeficiente de consanguinidad de los toros evaluados se utilizó el programa de cómputo Pedigree Viewer 5.5. El coeficiente de consanguinidad promedio fue de 3.6\% y el $68.2 \%$ de la población evaluada presentó una consanguinidad menor a 4\%. Además, se observó que el $41.2 \%$ de los padres de los machos evaluados eran de origen peruano y el $41 \%$ de lo abuelos maternos (padres de madres) eran también de origen peruano. El coeficiente de consanguinidad obtenido es relativamente bajo; sin embargo, es recomendable que los programas de mejora genética analicen el árbol genealógico de la vaca y el toro para controlar el coeficiente de consanguinidad en los hatos.

Palabras clave: consanguinidad, bovino, Brown Swiss, pedigrí

\section{Abstract}

Inbreeding can cause a depression in fertility performance, health and productivity of dairy cows and other animal husbandry species. The aim of this study was to estimate the inbreeding coefficient of Brown Swiss bulls registered in year 2003 and establish the origin of their sires and dam's sires. Records of 4 generations of 113 pedigree bulls registered in the Peruvian Brown Swiss Association in 2003 were analyzed, processing a total of 3616 pedigree certificates. The computer program Pedigree Viewer 5.5 was used to determine the inbreeding coefficient of these bulls. The average inbreeding coefficient was $3.6 \%$, and $68.2 \%$ of the analyzed population had an inbreeding coefficient of less

\footnotetext{
${ }^{1}$ Registros Genealógicos Zootécnicos del Perú, Programa de Mejoramiento Animal, Facultad de Zootecnia, Universidad Nacional Agraria La Molina, Lima.

Web: www.lamolina.edu.pe/registrosgenealogicos

${ }^{2}$ E-mail: emellisho@lamolina.edu.pe
} 
than $4 \%$. Besides, it was observed that $41.2 \%$ of the sire's sires evaluated had Peruvian origin and $41 \%$ of the dam's sires (maternal grandfather) had Peruvian origin too. The inbreeding coefficient obtained was relatively low, though, it is advisable that animal breeding programmes analyze the genealogical tree of the dam and sire in order to control inbreeding in herds.

Key words: inbreeding, bovine, Brown Swiss, pedigree

\section{INTRODUCCIÓN}

La consanguinidad es definida como la probabilidad que un animal herede los mismos alelos de sus padres, cuando tienen uno o más ancestros en común. Cuanto más cercanos y relacionados estén genealógicamente, esta probabilidad será más alta; sin embargo, solamente a través de una prueba con marcadores moleculares, es realmente posible conocer cuales son los alelos que cada animal hereda aleatoriamente de sus padres (Bhattacharya et al., 2003).

En ganadería lechera, los cálculos de consanguinidad han tomado importancia en esta última década debido a que se ha reportado un incremento progresivo de este valor, observándose animales consanguíneos que presentan depresión en el comportamiento productivo de su descendencia, ocasionando pérdidas económicas en la producción de leche (Cassell, 1984). Smith et al. (1998) indican que la producción acumulada por vida de leche decae en $790 \mathrm{lb}$ por cada incremento de $1 \%$ en la consanguinidad. Esto quiere decir que si, por ejemplo, una vaca Holstein tiene un coeficiente de consanguinidad de $6.25 \%$, la producción acumulada por vida será menor en $4937 \mathrm{lb}$. Asimismo, reportaron que los ingresos netos por vida disminuyen en US\$ 600.00 si un toro se cruza con su propia hija y en US\$ 300.00 si lo hace con su media hermana. Sin embargo, Lee (1998) afirma que los niveles de consanguinidad entre $5 \mathrm{y}$ $6 \%$, producen pérdidas económicas relativa- mente bajas y que niveles mayores pueden ser sobrellevados si el mérito genético del cruce es mayor a los valores esperados por la depresión en el comportamiento productivo y reproductivo.

Si bien el ganado lechero en la actualidad es mucho más consanguíneo, también es más productivo. Los productores no deben evitar el uso de los mejores hijos de un toro determinado simplemente porque tiene hijas en el rebaño, pues algunas combinaciones del toro con las vacas del rebaño pueden producir un nivel de endogamia con valores aceptables. Sin embargo, lo que sí debe evitarse son los cruzamientos que producen cifras inaceptables de endogamia, por los efectos negativos que esta ocasiona (Cassell, 1984).

Las estimaciones de coeficiente de consanguinidad usando dos o tres generaciones de pedigrí, siempre serán menores que los basados en datos de diez o veinte generaciones (Van Doormaal, 2008). Sin embargo, Cassell (1984) sostiene que la información completa de cuatro o cinco generaciones es suficiente para obtener datos precisos de consanguinidad.

En el Perú, no existe información relacionada con los niveles de consanguinidad en ganado lechero; por lo que el presente estudio tuvo como objetivo estimar el coeficiente de consanguinidad de los toros de la raza Brown Swiss registrados en la Asociación Brown Swiss del Perú, y establecer el origen de los padres y abuelos maternos. 


\section{Materiales y Métodos}

Se empleó los certificados de pedigrí de los animales machos registrados en la Asociación Brown Swiss del Perú el año 2003. Se evaluó la consanguinidad de 113 animales con información completa de 4 generaciones antecesoras, dentro de un total de 170 toros registrados distribuidos entre 30 criadores; asimismo, se evaluó el origen de los padres y de los abuelos en el total de la población registrada en el 2003.

Para la determinación del coeficiente de consanguinidad de los toros evaluados se utilizó el programa de cómputo Pedigree Viewer 5.5, desarrollado por Brian y Sandy Kinghorn, Universidad de New England, Australia (http:/ /www-personal.une.edu.au/ bkinghor/ pedigree.htm).

Se realizó una matriz en una hoja de cálculo de Excel 2003, donde se colocó la información de la genealogía de cada toro, así como de sus cuatro generaciones ascendentes, y la información del origen de sus padres y abuelos maternos. Con los datos de la genealogía, se creó un archivo en el programa Bloc de Notas, requerido por el programa Pedigree Viewer. Ese archivo .PED consta de tres columnas: la identidad del individuo, la identidad del padre y la identidad de la madre.

El método que utiliza Pedigree Viewer para el cálculo del coeficiente de consanguinidad, es el desarrollado por Wright (1922), citado por Lush (1969), siendo la fórmula:

$\mathrm{F}_{\mathrm{x}}=0.5^{\mathrm{n} 1+\mathrm{n} 2+1}\left(1+\mathrm{F}_{\mathrm{A}}\right)$

donde,

$\mathrm{F}_{\mathrm{x}}=$ coeficiente de consanguinidad del animal $\mathrm{x}$

$\mathrm{n}_{1}=$ número de generaciones que va desde un padre al antecesor común

$\mathrm{n}_{2}=$ número de generaciones desde el otro padre al antecesor común

$\mathrm{F}_{\mathrm{A}}=$ coeficiente de endogamia del antecesor A
El programa construye un diagrama de flechas del pedigrí en el que identifica cada individuo y diferencia por colores las líneas paternas y maternas. Se procedió a establecer el promedio general y por criador. Además, se calculó la desviación estándar y el coeficiente de variación de los valores de consanguinidad de los toros.

Para definir el origen de los padres y abuelos maternos se utilizó la matriz en Excel con los datos de genealogía, utilizando la función de filtro para la columna en donde se detallaba el país de origen para facilitar su cuantificación y posteriormente expresar el resultado en porcentaje.

\section{Resultados y Discusión}

Se procesó 3616 certificados de registro de pedigrí para 113 toros en cuatro generaciones, obteniéndose una consanguinidad de 3.6\%. Resultados publicados para vacunos Brown Swiss nacidos en el 2007 en EEUU señalan niveles de consanguinidad de 5.8\% (Lawlor et al., 2007). Esto podría deberse a que en el Perú, el uso de toros registrados bajo el sistema de monta natural es más frecuente que el uso de semen congelado en inseminación artificial, lo que conlleva a una selección menos intensa de los reproductores. Cassell (1984) argumenta que valores elevados de endogamia se obtienen como consecuencia de usar solo unos cuantos toros en los diferentes programas de mejoramiento.

El rango de endogamia obtenido varía entre 0 a $23.4 \%$, donde el $85.8 \%$ de los animales evaluados presentó valores de consanguinidad inferiores a $6 \%$. La importancia de estos resultados radica en que valores de consanguinidad superiores a $5-6 \%$ producen pérdidas económicas significativas por la depresión del comportamiento de las hijas (Lee, 1998). La varianza, desviación y coeficiente de variación obtenidos fueron de 15.4, 3.9 y $106.5 \%$, respectivamente, aparentemente 
Cuadro 1. Distribución del coeficiente de consanguinidad de toros Brown Swiss registrados en la Asociación Brown Swiss del Perú en el 2003

\begin{tabular}{ccc}
\hline $\begin{array}{c}\text { Niveles de consanguinidad de } \\
\text { toros }(\%)\end{array}$ & $\begin{array}{c}\text { Ganaderos } \\
\mathrm{n}(\%)\end{array}$ & $\begin{array}{c}\text { Animales } \\
\mathrm{n}(\%)\end{array}$ \\
\hline $0-2$ & $11(36.7)$ & $44(39.0)$ \\
$2-4$ & $9(30.0)$ & $33(29.2)$ \\
$4-6$ & $6(20.0)$ & $20(17.7)$ \\
$6-8$ & 0 & $6(5.3)$ \\
$8-10$ & 0 & $1(0.9)$ \\
$10-12$ & $2(6.7)$ & $1(0.9)$ \\
$12-14$ & 0 & $2(1.8)$ \\
$14-16$ & $1(3.3)$ & $5(4.4)$ \\
$>16$ & $1(3.3)$ & $1(0.9)$ \\
\hline
\end{tabular}

Cuadro 2. Distribución del origen de los padres y los abuelos maternos de los toros Brown Swiss registrados en la Asociación Brown Swiss del Perú en el 2003

\begin{tabular}{lcc}
\hline País de origen & $\begin{array}{c}\text { De padres } \\
\mathrm{n}(\%)\end{array}$ & $\begin{array}{c}\text { De abuelo materno } \\
\mathrm{n}(\%)\end{array}$ \\
\hline EEUU & $72(42.4)$ & $51(30.7)$ \\
Perú & $70(41.2)$ & $68(41.0)$ \\
Canadá & $15(8.8)$ & $45(27.1)$ \\
Alemania & $11(6.5)$ & $2(1.2)$ \\
Italia & $2(1.2)$ & 0 \\
\hline
\end{tabular}

debido a que, en muchos casos, los ganaderos no consideraron la consanguinidad como criterio de selección para los apareamientos.

La gran mayoría de criadores $(86.7 \%)$ tuvo promedios de consanguinidad por debajo del valor máximo aconsejable $(6 \%)$. No obstante, cuatro criadores obtuvieron valores elevados de endogamia (Cuadro 1). El origen de los padres de los toros Brown Swiss registrados fue principalmente americano y peruano, mientras que en el caso de los abue- los maternos fue peruano, americano y canadiense. El aporte de toros italianos y alemanes no fue significativo para este estudio (Cuadro 2).

Se puede emplear animales con niveles de consanguinidad mayores a los recomendados en los diversos programas de mejoramiento, si se va a aparear con ejemplares con los que no tengan ningún grado de parentesco o si el mérito genético del cruce es mayor a los valores esperados por la depresión en el 
comportamiento productivo. Es importante conocer el coeficiente de consanguinidad de todo animal que nace en el hato, ya que permite tener un mayor criterio de selección, en especial en aquellos hatos ganaderos que usan sus propios toros como reproductores.

\section{Conclusiones}

El valor de consanguinidad promedio de toros Brown Swiss registrados en la Asociación Brown Swiss del Perú en el 2003 fue relativamente bajo (3.6\%).

\section{Literatura Citada}

1. Bhattacharya T, Kumar P, Kumar S. 2003. Estimation of inbreeding in cattle using RAPD markers. J Dairy Res 70: 127-129.

2. Cassell B. 1984. Inbreeding. Virginia Tech Cooperative Extension [Internet], [mayo 1999]. Disponible en: http:// www.ext.vt.edu/pubs/dairy/404-080/ 404-080.html
3. Lawlor T. 2007. Inbreeding has slowed down. Holstein Pulse [Internet], [junio 2007]. Disponible en: http:// www.holsteinusa.com/pdf/pulse/ pulse_2007_fall.pdf

4. Lee K. 1998. Managing inbreeding in your breeding program. Michigan Dairy Review [Internet], [febrero 1998]. Disponible en: http://www.canr.msu.edu/ dept/ans/vol3no1.htm

5. Lush J. 1969. Bases para la selección animal. Buenos Aires: Ed. Agropecuarias Peri. 673 p.

6. Smith L, Cassell B, Pearson R. 1998. The effects of inbreeding on lifetime performance of dairy cattle. J Dairy Sci 81: 2729-2737.

7. Van Doormaal B. 2008. Demystifying inbreeding. Canadian Dairy Network [Internet], [enero 2008]. Disponible en: h t t p : / / w w w . c d n . c a / document.php?id=143

8. Wiggans G, Van Raden P, Zuurbier J. 1995. Calculation and use of inbreeding coefficients for genetic evaluation of United States dairy cattle. J Dairy Sci 78: 1584-1590. 